\title{
Sport and exercise headache: Part 2. Diagnosis and classification
}

\author{
Stephen J. Williams BMed Sci and Hitoshi Nukada MD \\ Department of Medicine, University of Otago Medical School, Dunedin, New Zealand
}

\begin{abstract}
A group of 129 subjects (67 men and 62 women) experiencing sports headache was established using a questionnaire. A wide range of information was gathered, focusing on the mode of onset, time course, characteristic features and associated symptoms of sports-related headache. Criteria for the varieties of sports headaches were established using head trauma and then migraine to divide subgroups of sports headaches. Cases were classified into four categories: effort migraine, traumatriggered migraine, effort-exertion headache, and posttraumatic headache. The percentages of each sportsrelated headache found were: effort migraine $9 \%$, traumatriggered migraine $6 \%$, effort-exertion headache $60 \%$, post-traumatic headache $22 \%$ and miscellaneous $3 \%$. Sports migraine accounted for $15 \%$ of the total sports headache sample. Effort-exertion headache was the most common type of sports headache. Although effort-exertion headache could be separated into subjects who had an acute severe headache induced by anaerobic exercise (exertion headache) from those having a substantial headache lasting hours initiated by aerobic exercise (effort headache), most subjects with effort-exertion headache in this study appeared not to fall into any discrete subgroups. Trauma-related headaches were experienced mainly by men in contact sports, while women more commonly had non-trauma-related headache in running and jogging.
\end{abstract}

Keywords: Effort-exertion headache, effort migraine, post-traumatic headache, sports headache, traumatriggered migraine

The prevalence of sport and exercise headache among university students was assessed using a questionnaire, and we found that approximately one-third of them had experienced sports-related headache ${ }^{1}$. In this part, we classified those headaches into each subgroup of sports headaches. A major concern in headache epidemiology is that of definition of each group of headache. Attempts to classify headaches are numerous, and both the Ad Hoc Committee on Classification of Headache of the National Institute of Neurological Diseases and Blindness and the Headache Classification Committee of the International Headache Society divided

Address for correspondence: Dr Hitoshi Nukada, Department of Medicine, University of Otago Medical School, PO Box 913,

Dunedin, New Zealand

(C) 1994 Butterworth-Heinemann Ltd

0306-3674/94/020096-05 headaches into two major groups: vascular headache of migraine type and muscle contraction or tension headache ${ }^{2,3}$. There is still a great deal of debate as to whether these two forms of headaches are separate clinical entities. Some believe the two to be opposing ends of a spectrum within which most recurrent headaches fall. For example, in any individual subject some attacks fit in with classical migraine and some with tension headache, posing a problem for headache division ${ }^{3}$. The pathophysiological mechanism of migraine is still controversial ${ }^{4}$. Many theories and hypothetical models exist to explain the complex series of biochemical, pharmacological and neurovascular events that characterize the migraine attack.

Sport and exercise headaches have been reported only as a series of individual cases, and distinct forms of sport- and exercise-related headache have been described on the basis of their characteristics and precipitating events. It is generally accepted to label headaches by individual characteristics, although the direct cause of headache has often been used to categorize a headache such as 'footballer's migraine' or 'weight-lifter's headache'. We investigated the characteristic features of sports headache in 129 subjects, and set diagnostic criteria to classify their sports headaches into each subgroup. Since sports headache is a direct result of exercising or playing sport, head trauma is a major precipitating factor in the cause of headache, providing a clear distinction between headache groups. The trauma and nontrauma groups are further divided into migraine and non-migraine groups.

\section{Methods}

In addition to 103 university students experiencing sports headache found in Part 1 of this study ${ }^{1}, 26$ subjects (17 men and 9 women) who were also suffering from sports headache were drawn from no particular population, making a total of 129 subjects. The latter group consisted of 23 students and three non-students who completed the questionnaire while visiting the Otago University Health Centre. The overall group consisted of 67 men $(52 \%)$ and 62 women $(48 \%$ ) with a mean(s.d.) age of $20.7(3.3)$ (range 15-40) years. Diagnosis and classification of sports-related headache in individual cases were based on the evaluation of a wide range of information gathered by the questionnaire. To establish set criteria for sport and exercise headache, 
we reviewed questionnaires completed by 129 sports headache sufferers, and the sports headache literature, focusing on the cause, medical history and characteristic symptoms.

The division of sports headache created four basic groups using head trauma and migraine as the basis for division. Eight criteria for sports-related migraine are listed using the definition of migraine with aura by the International Headache Society. The first three criteria must be fulfilled for a headache to be classified as a sports-related migraine unless a number of other factors clearly establish the headache as sports migraine: (1) a visual or sensory warning or any other aura before the onset of headache; (2) headache lasting for several hours; (3) at least two episodes of the headache have been experienced; (4) unilateral location of the headache; (5) pounding or throbbing severe headache; (6) nausea and vomiting associated with headache; (7) a past history of non-sports related migraine; and (8) a family history of migraine. Diagnosis of sports migraine creates two headache varieties: (1) effort migraine - sports migraine initiated by physical effort, often associated with a number of aggravating factors such as dehydration, extreme exercise, heat, altitude and hypoglycaemia ${ }^{5,6}$; and (2) trauma-triggered migraine - sports migraine initiated by mild head trauma ${ }^{7-12}$.

Non-migrainous headaches without head trauma were classified into effort-exertion headache or the miscellaneous group. Although post-traumatic nonmigraine headache is not a clearly defined group, chronic post-traumatic headache, which includes the majority of headaches following head trauma, was not considered in the present study ${ }^{1,13}$

\section{Results}

Diagnosis of headache varieties through the diagnostic tree is shown in Figure 1. Of the 129 sports headaches $92(71 \%)$ were not associated with head trauma, and were classified into effort migraine in 11 subjects, effort-exertion headache in 77 and miscellaneous headaches in four. Headaches in 37 subjects occurred as a result of head trauma, and eight of them were diagnosed as trauma-triggered migraine, while the other 29 were classified as post-traumatic headache.

\section{Effort migraine}

Effort migraine was diagnosed in 11 subjects (Figure 1) eight $(73 \%)$ of whom were women (mean(s.d.) age $21.5(1.1)$ years). Effort migraine contributed to $13 \%$ of sports headaches in women and $5 \%$ in men. It was initiated by a variety of sports, particularly running and jogging (six subjects). Extreme exercise in hot conditions was cited as provoking migraine in two subjects. One subject noted migraine from an intense session of weight-training, indicating that exertion as well as activity requiring effort could initiate migraine. Other sports causing effort migraine include netball, hockey, cycling and swimming. The migraines were experienced only occasionally by nine of the subjects, but two cases had migraine on every

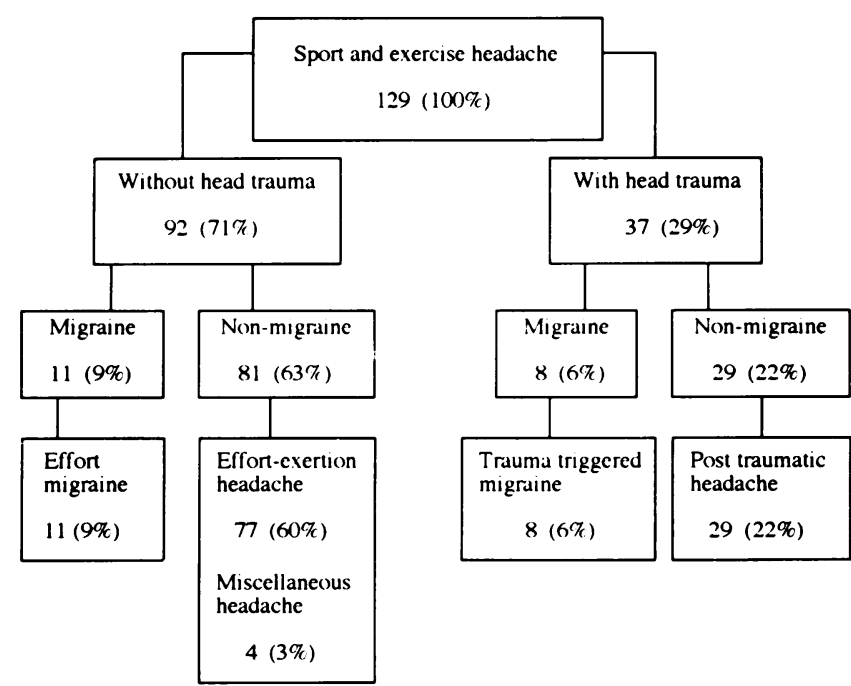

Figure 1. Classification of 129 sports-headache subjects

second and third occasion that they participated in the particular exercise. Effort migraine began in childhood or adolescence, with an average age of onset of 15 (range 10-21) years. Aura was noticed by all, $45 \%$ experienced vomiting, $82 \%$ nausea and $45 \%$ stiffness in the neck. The migraine was generally described as having a throbbing/pounding character. The average migraine was described as either severe or moderate and $50 \%$ of the subjects reported that migraine affected their social life in some ways. Five subjects recovered within hours and the remaining six after a night's sleep. Spontaneous migraine which did not relate to sport or exercise was experienced in $55 \%$ of subjects, with a family history present in $64 \%$. Both of these figures were higher when compared with those among the trauma-triggered migraine subjects.

\section{Trauma-triggered migraine}

Diagnosis of trauma-triggered migraine (TTM) was made in eight subjects (Figure 1), six men and one woman (mean(s.d.) age 23.6(4.9) years). Unlike effort migraine, which was predominantly experienced by women, TTM was experienced mostly by men $(88 \%)$, contributing $10 \%$ of sports headache in men compared with only $1.5 \%$ in women. TTM was initiated during contact sports in all subjects with the exception of one case (cricket). Head trauma initiating TTM was mild, with none of the subjects reporting any loss of consciousness. TTM was seen in the first two decades of life, often emerging in childhood (average age of TTM onset being 13 years). TTM displayed a number of clinical features in common with classical migraine. All had an aura, comprising visual and/or sensory disturbances before the headache, which was followed by severe headache accompanied by nausea $(75 \%)$ and vomiting $(25 \%)$. The headache was described as having a throbbing/ pounding nature, commonly located in the forehead. The duration of headache was several hours, lasting all day for two subjects. By $24 \mathrm{~h}$ all the subjects had fully recovered. Six subjects $(75 \%)$ experienced 
repeated episodes with head trauma. Spontaneous migraine without sports or exercise was noted in $25 \%$ of subjects, and $38 \%$ of subjects had a family history of migraine.

\section{Effort-exertion headache}

Effort-exertion headache (EEH) was the most common type of sports headache (Figure 1). Some form of $\mathrm{EEH}$ was experienced by 31 men and 46 women contributing to $74 \%$ of the sports headaches in women and $46 \%$ in the men. A variety of sports and exercises were described as initiating $\mathrm{EEH}$, running and jogging being most common (Table 1). For the majority the headache developed over minutes or hours, although a small proportion experienced the headache occurring within seconds. The duration of headache ranged from $5 \mathrm{~min}$ to $24 \mathrm{~h}$. EEH could be divided into two categories: an acute headache lasting seconds or minutes with rapid recovery (exertion headache) and a gradual headache lasting an hour or longer (effort headache). From 38 subjects reporting aggravating factors, three men suffered from headache for seconds or minutes when attempting particularly strenuous weight training. Rapid exertion and anaerobic effort such as hard sprinting induced an acute headache in five subjects. In comparison, subjects listing factors established over a longer time frame, such as dehydration, heat, tiredness and endurance exercise, almost unanimously experienced a headache lasting an hour or longer. These long-lasting headaches all involved aerobic effort. However, the difficulty in classifying EEH can be seen by looking at the 27 subjects whose headaches were induced by running. The duration of the headache is an hour or more for 14 and minutes for the remaining 13 subjects. Eleven were able to give aggravating factors ranging from jarring producing a headache lasting minutes to being fatigued and developing a headache lasting hours.

$\mathrm{EEH}$ was experienced only occasionally by most subjects, with a small number experiencing headache on every occasion or every second or third occasion.

Table 1. Sports initiating effort-exertion headache in 77 subjects

\begin{tabular}{|c|c|c|c|c|}
\hline \multirow[t]{2}{*}{ Sport initiating EEH } & \multicolumn{2}{|c|}{ Men* } & \multicolumn{2}{|c|}{ Woment } \\
\hline & No. & $\%$ & No. & $\%$ \\
\hline Running/jogging & 13 & 37 & 14 & 29 \\
\hline Aerobics & - & - & 7 & 15 \\
\hline Weights/gym exercises & 6 & 17 & 3 & 6 \\
\hline Cycling & - & - & 3 & 6 \\
\hline Swimming & 2 & 6 & 4 & 9 \\
\hline Rugby & 4 & 11 & 3 & 6 \\
\hline Any extreme exercise & 2 & 6 & 1 & 2 \\
\hline Hockey & - & - & 3 & 6 \\
\hline All sports & - & - & 2 & 4 \\
\hline Others $¥$ & 8 & 23 & 5 & 11 \\
\hline Sport not mentioned & - & - & 2 & 4 \\
\hline
\end{tabular}

${ }^{*} n=31 ;+n=46 ; \ddagger$ Variety of sports initiating headache of two or less in number.
The pain was described as either aching/dull or pounding/throbbing. The location of the pain was variable - 'forehead' or 'generalized' were the most common descriptions. EEH was not as severe as other sports headache varieties, particularly when compared with sports migraine. None of the headaches was described as severe; the average headache was scored either mild $(48 \%)$ or moderate $(52 \%)$. This was reflected in the infrequency of $\mathrm{EEH}$ subjects receiving medical attention, and only two were seen by a doctor for their sports headache. Loss of concentration was the most common associated symptom, and nausea and neck stiffness were also commonly reported. Approximately a quarter of 74 subjects had some form of warning symptoms before the onset of headache, but failed to meet the other criteria for migraine, for example lasting only a few minutes. A positive past history of spontaneous migraine was found in $9 \%$ and a positive family history of migraine in $14 \%$.

\section{Post-traumatic headache}

Headaches due to head trauma, but not traumatriggered migraine, occurred in 29 subjects and were classified as post-traumatic headache (Figure 1). Like trauma-triggered migraine, it was the result of head trauma in a contact sport and therefore was more prominent in men, comprising 23 men (accounting for $34 \%$ of sports headache in men) and six women (accounting for $10 \%$ of sports headache in women). The head trauma initiating post-traumatic headache was regarded as minor, but five of the subjects reported short-term loss of consciousness in association with their headaches. Confusion and loss of concentration were common associated symptoms. Eleven subjects reported stiff neck with posttraumatic headache, suggesting neck hypertension occurring with head trauma. The duration of this type of headache varied from minutes to all day. Although the average severity was described as moderate, seven subjects were unable to continue the sport or exercise because of severe headache. The pain was generally described as either aching/dull or throbbing/pounding, and was usually a non-localized headache.

A number of post-traumatic headache subjects showed susceptible migrainous features. Nine had a family history of migraine, and three suffered from spontaneous migraine. A case of a 15 -year-old boy is of particular interest. He appeared to have traumatriggered migraine, experiencing numbness and tingling followed by a severe pounding headache, but lasting only for several minutes. Furthermore, his father was diagnosed as suffering from traumatriggered migraine.

Four subjects $(3 \%)$ were placed in the category of miscellaneous sports headache. In each subject, there was a clear distinguishing factor responsible for headache. A brief description is given in two cases: (1) a woman experienced a brief headache only when wearing a protective helmet during hockey matches; and (2) a wheelchair road racer experienced a headache due to a full bladder, and relief was easily obtained by draining the bladder. 


\section{Discussion}

Sports headaches were classified into four major groups: classical migraine, with or without headache trauma (trauma-triggered migraine and effort migraine); and non-migrainous headache, with or without trauma (post-traumatic headache and effort-exertion headache). A major problem in headache epidemiology is that of definition and classification. In any individual subjects some attacks fit in with classical migraine, some with common migraine, and some with tension headache posing a problem for headache division ${ }^{14}$. To date no unanimously approved criteria exist for diagnosing migraine or other headache varieties, especially when the information is gathered by questionnaire ${ }^{15}$. As we defined migraine on the basis of classical attacks, if common migraine is included the prevalence of sports-related migraine could be greater.

Sports migraine consists of two varieties: effort migraine and trauma-triggered migraine. Effort migraine has not been well documented in the literature. As migraine in general is more common among women, effort migraine in the present study is predominantly experienced by women. Among subjects experiencing effort migraine, a high percentage suffered from non-sports related migraine and gave a positive family history of migraine. Although the pathogenesis of effort migraine is still unidentified, migraine triggered by physical effort was particularly evident among highly trained athletes in the 1968 Summer Olympic Games in Mexico City (altitude 7000 feet above sea level) suggesting that low oxygen tension may trigger migraine by an as yet unknown mechanism ${ }^{16,17}$. At sea level effort migraine is rarely seen in élite athletes.

Matthews was the first to describe subjects with trauma-triggered migraine, which he aptly named 'Footballer's migraine'7. He reported three soccer players and a boxer who had classical migraine resulting from a blow to the head. Since then a number of cases of trauma-triggered migraine have been documented ${ }^{8-12,18}$, and the information gathered from eight cases of trauma-triggered migraine in the present study was consistent with those reported. The migraine is initiated by mild head trauma, and is predominantly experienced by men. The head trauma is not necessarily heavy; a light blow to the head or face can trigger migraine ${ }^{9}$. The clinical picture of trauma-triggered migrain appears identical to that of classical migraine. Warning symptoms were usually present, either visual disturbances, e.g. scintillating scotoma, flashing lights and blurred vision, or sensory symptoms, e.g. tingling or numbness of a portion of the body, and an unusual feeling in the stomach. Nausea and vomiting were commonly associated with a severe throbbing headache lasting for several hours. A past history of non-sports-related migraine and a positive family history of migraine appear to be dominant factors ${ }^{19}$, but occurred less frequently than expected in the current study. Trauma-triggered migraine is predominantly experienced by children or adolescents, usually beginning between the ages of 10 and 25 years. The aetiology of migraine after head trauma is still obscure and the possible long-term sequelae of this 'benign' condition are unknown.

Effort-exertion headache is the most common and most diverse of the subgroups of sports headache. Effort-exertion headache comprises approximately three-quarters of women's sports headaches and less than half of men's. Although a variety of sports and exercises initiated effort-exertion headaches, running and jogging made the largest contribution. The distinction between effort and exertion headaches in the literature is confusing, although tentative criteria can be established as follows. Exertion headache appears to be induced by particular exercises such as strenuous lifting, bending over and physical jarring, and is often associated with pain in the occipital and neck region lasting only minutes ${ }^{20,21}$. Effort headache lasting several hours is initiated by aerobic exercise and may be aggravated by dehydration, heat, fatigue and extreme exercise ${ }^{5,6}$. However, some authors have suggested that separation of these two headache types is unnecessary; both have features in common with altitude headache ${ }^{6}$.

The term 'benign exertional headache' has been suggested by Rooke $^{20}$, and exertional headaches are a direct result of some form of exertion: lifting, running, coughing ('cough headache'), sneezing, sexual intercourse ('coital headache'). They are generally headaches with a rapid onset and brief duration, occurring in all parts of the head. There is some variation among the literature regarding the duration of exertion headache, ranging from minutes to all day. Diamond reported 15 cases as 'prolonged benign exertion headache' with a mean duration of $4 \mathrm{~h}^{22}$. The headache was initiated by physical exertion in five cases, with the remainder from coughing or stooping. Paulson reported a case of 'weight-lifter's headache in which a 21-year-old suffered a headache upon lifting weights, but also when sneezing or upon orgasm $^{23}$. A number of possible underlying mechanisms of exertion headache have been postulated: raised intrathoracic pressure ${ }^{24,25}$, compressed blood vessels by muscle tension ${ }^{25,26}$, vasodilation of cerebral blood vessels by stress ${ }^{14}$, neck muscle tension or strain $^{23}$, neurogenic, e.g. stimulating the monoaminergic pathway ${ }^{27}$ or a combination of these factors $^{28}$. In the case of exertion headache in luge athletes, multiple factors my be involved in its development such as continued neck flexion and strain, the exertion of racing, altitude, and the body position adopted to counter gravity ${ }^{27}$. A number of factors appears to aggravate effort headaches: altitude, dehydration, heat, hypoglycaemia, poor fitness, extreme exercise, and even alcohol and caffeine consumption $5,6,29$. Although some effort-exertion headaches may be the result of one particular factor, it is more probably that several factors are involved. Other forms of headache, such as tension headache, might also be responsible for a headache experienced during exercise $\mathrm{e}^{30}$.

The category of post-traumatic headache is not clearly demarkated and the symptoms vary. Posttraumatic headache is usually due to a substantial blow to the head, face or neck, and therefore is predominantly found in contact sport. The headache is of variable intensity and location. The severity of 
trauma has little relationship to the intensity of headache and, if anything, the more severe the trauma the less the degree of headache experienced $^{31}$. Post-traumatic headache is often associated with concussion, and headache is a common symptom in mild concussion ${ }^{32}$. Post-traumatic headache is often overlooked, since headache with or without concussion is not taken seriously by the individual, trainer or peer group ${ }^{25}$. Lombardo and McKeag described a 16-year-old footballer who continued to play despite repeated headaches after head trauma, because he was afraid of being prevented from playing ${ }^{33}$. It is important to have the headache assessed, particularly when other symptoms, e.g. disturbance of consciousness, memory impairment, vomiting, dizziness, weakness or sensory complaints, are associated with headache, or when headaches occur on more than one occasion.

\section{Acknowledgements}

We are grateful to Brian Niven for his assistance in statistical analysis, Dave Gerrard and Mike Flanagan for their advice and discussion, and the staff of the Medicine Department for their help. S.J.W. was supported by the Health Research Council of New Zealand.

\section{References}

1 Williams S, Nukada H. Sport and exercise headache: Part 1. Prevalence among university students. Br J Sports Med 1994; 28: 90-5.

2 Ad Hoc Committee on Classification of Headache. Classification of headache. JAMA 1962; 179: 717-18.

3 Headache Classification Committee of the International Headache Society. Classification and diagnostic criteria for headache disorders, cranial neuralgias and facial pain. Cephalalgia 1988; 8(Suppl 7): 1-96.

4 Moskowitz MA. Neurogenic versus vascular mechanisms of sumatriptan and ergot alkaloids in migraine. Trends Pharmacol Sci 1992; 13: 307-11.

5 Dalessio DJ. Effort migraine. Headache 1974; 14: 53.

6 Massey EW. Effort headache in runners. Headache 1982; 22 99-100.

7 Matthews WB. Footballer's migraine. BMJ 1972, 2, 326-327.

8 Espir, MLE, Hodge ILD and Matthews PHN. Footballer's migraine. BMJ 1972; 2: 352.

9 Morris, AM. Footballer's migraine. BMJ 1972; 2: 769-70.

10 Kalenak A, Petro DJ, Brennan RW. Migraine secondary to head trauma in wrestling. Am I Sports Med 1978; 6: 112-13.

11 Bennett DR, Fuenning SI, Sullivan G, Weber J. Migraine precipitated by head trauma in athletes. Am J Sports Med 1980; 8: $202-5$

12 Ashworth B. Migraine, head trauma and sport. Scott Med J 1985; 30: 240-2.

13 Friedman AP. Headache. In: Baker AB, Baker LH, eds. Clinical Neurology, Vol 2. Philadelphia, Pennsylvania, USA: Harper and Row, 1983; 13: 1-50.

14 Rose CF. Headache: definitions and classification. In: Vinken PJ, Bruyn GW, eds. Handbook of Clinical Neurology Vol 48, Amsterdam, Netherlands: Elsevier, 1986: 1-12.

15 Nikiforow R, Hokkanen E. An epidemiological study of headache in an urban and a rural population in northern Finland. Headache 1978; 18: 137-45.

16 Atkinson R, Appenzellar O. Headache in sport. Seminars in Neurology 1981; 1: 334-43.

17 Jokl E. Olympic medicine/sports cardiology. Ann Sports Med 1984; 1: 148-9.

18 Shadel RF, Puffer JC. Managing traumatic headaches in a football player. Physician and Sportsmedicine 1990; 18: 81-4.

19 Haas DC, Lourie H. Trauma-triggered migraine: an explanation for common neurological attacks after mild head injury. J Neurosurg 1988; 68: 181-8.
20 Rooke ED. Benign exertional headache. Med Clin North Am 1968; 52: 801-8.

21 Perry WJ. Exertional headache. Physician and Sportsmedicine 1985; 13: 95-9.

22 Diamond S. Prolonged benign exertional headache: its clinical characteristics and response to indomethacin. Headache 1982 22: 96-8.

23 Paulson GW. Weightlifters headache. Headache 1983; 23: 193-4.

24 Lambert RW Jr, Burnet DL. Prevention of exercise induced migraine by quantitative warm-up. Headache 1985; 25: 317-19.

25 McCarthy P. Athletes' headaches: not necessarily 'little' problems. Physician and Sportsmedicine 1988; 16: 169-73.

26 Cleveland $\mathrm{H}$. Headaches: a weighty problem for lifters? Physician and Sportsmedicine 1984; 12: 23.

27 Roos R. Luge participation is hard on the head. Physician and Sportsmedicine 1986; 14: 185-8.

28 Diamond S, Solomon GD, Freitag FG. Headache in sport. In: Jordan BD, Tsairis P, Warren RF eds. Sports Neurology. Rockville, Maryland, USA: Aspen, 1989: 127-32.

29 Thompson JK. Exercise-induced migraine prodrome symptoms. Headache 1987; 27: 250-1.

30 Garfinkel D. Headache in athletes. Physician and Sportsmedicine 1983; 11: 67-76.

31 Kelly RE. Post-traumatic headache. In: Vinken PJ, Bruyn GW eds. Handbook of Clinical Neurology Vol 48, Amsterdam, Netherlands: Elsevier, 1986: 383-90.

32 Nelson WE, Jane JA, Gieck JH. Minor head injury in sports: a new system of classification and management. Physician and Sportsmedicine 1984; 12: 103-7.

33 Lombardo JA, McKeag DB. Football player with a persistent headache. Physician and Sportsmedicine 1987; 15: 75-9. 